\title{
The role of nesfatin and selected molecular factors in various types of endometrial cancer
}

\author{
Anna Markowska ${ }^{1}$, Monika Szarszewska ${ }^{2}$, Pawel Knapp ${ }^{3}$, Anna Grybos ${ }^{4}$, Marian Grybos ${ }^{5}$, \\ Andrzej Marszalek ${ }^{6}$, Violetta Filas ${ }^{6}$, Katarzyna Wojcik-Krowiranda ${ }^{7}$, Malgorzata Swornik ${ }^{8}$, \\ Janina Markowska² \\ ${ }^{1}$ Department of Perinatology and Gynaecology, Poznan University of Medical Sciences, Poland \\ ${ }^{2}$ Department of Oncology, Gynaecological Oncology, Poznan University of Medical Sciences, Poland \\ ${ }^{3}$ Department of Gynaecology and Gynaecologic Oncology, Medical University of Bialystok, Poland \\ ${ }^{4}$ Department of Gyneacology and Obstetrics, Faculty of Health Science, Wroclaw Medical University, Poland \\ ${ }^{5}$ Medical School in Opole, Poland \\ ${ }^{6}$ Department of Tumour Pathology and Prophylaxis, Poznan University of Medical Sciences, Greater Poland Cancer Centre, \\ Poznan, Poland \\ ${ }^{7}$ Medical University of Lodz, Poland \\ ${ }^{8}$ Gynaecological Endocrinology Department, Jagiellonian University Medical College, Cracow, Poland
}

\begin{abstract}
Objectives: Endometrial cancers (ECs) are the most common gynaecological cancers in well developed countries. Diabetes and metabolic syndrome are among the biggest risk factors. Nesfatin-1, the adipokine derivative of NUCB2 (nucleobindin derivative 2) is linked to the clinical course of EC. Molecular factors, including mutations in MLH1 and MHS2 genes, c-MET and ARID1A are also related to prognosis in endometrial cancer.

Material and methods: Using sections of paraffin-embedded preparations and immunohistochemistry, the expression of NESF1, MLH1, MSH2,C-MET and ARID1A were examined.

Results: In this study on protein expression, EC tissues manifested (although insignificantly) an elevated expression of NESF-1 in type II EC. In type I EC, NESF-1 expression was significantly higher in G1 in comparison to G2 and G3 together. A significantly lower expression of MLH1 was demonstrated in type I EC.

Conclusions: The most pronounced expression involved c-MET in all EC I and EC II tissues (in over $80 \%$ of cases). A tendency was detected for a high expression of NESF-1 in patients with type II EC, who also exhibited a high expression of MSH2.

Key words: endometrial cancer; NESF-1; MLH1; MSH2; c-MET; ARID1A
\end{abstract}

Ginekologia Polska 2019; 90, 10: 571-576

\section{INTRODUCTION}

Endometrial cancer $(\mathrm{EC})$ is the most commonly occurring gynaecological malignancy in economically developed countries. In Europe, the incidence rate amounts to 13.6 per 100.000 women. In many European countries an increased incidence of this tumor has been noted since 2005 [1-3]. In over $90 \%$ of EC cases, the cancer develops after the age of 50 , with a median age of 63 ; in $10-15 \%$ it is diagnosed before the age of $45[2,4]$.

Bokhman's hypothesis led to the identification of two types of EC differing in etiology, biology and clinical course [5].
Type I EC (endometrioid adenocarcinoma) involves 80\% of all EC cases. In most cases it is sporadic and linked to unbalanced estrogen stimulation and metabolic syndrome. This EC type manifests slow clinical course and positive prognosis. This type of cancer contains mutations in the PTEN, KRAS, CTNNB1, P1K3CA genes and in mismatch repair (MMR) genes, typical for Lynch syndrome [2, 6-8].

Type II EC (non-endometrioid adenocarcinoma) histologically encompasses serous, clear cell, poorly differentiated cancers of aggressive biology and an unfavorable clinical course. They contain mutations in TP53, HER2-neu and BRCA $[2,9,10-12]$. 
Type I EC with a high histological grade (G3) manifests a similarly poor prognosis to uterine serous carcinoma (USC), belonging to type II $[6,3,13,14]$, some well-differentiated, early diagnosed endometrioid EC gives recurrent disease, which suggests that the traditional Bokman's division may not be up to date.

Recent results have shown that more EC types exist. The heterogeneity of ECs has been proven by genomic analysis of endometrioid and serous cancers. The Cancer Genome Atlas (TCGA) developed in 2013, indicates there are 4 types of ECs [15]:

- unique nucleotides spectrum ultra-mutated tumors (POLE) - associated with favourable outcome,

- highly-mutated with microsatellite instability, mostly with MLH1 promoter methylation,

- a group of low frequency of mutations with low gene copy numbers,

- a group with high gene copy numbers and a low frequency of mutations, mainly serous cancers, connected to a poor prognosis.

It is suggested that the Genomic Atlas classification (TGGA) should be linked to the histological classification of ECs, particularly ECs of poor differentiation [16].

The acknowledged and recognized factors which affect the clinical course and type of oncological treatment of EC include the histological type, grading $(\mathrm{G})$ and clinical stage of disease. However, these traditional prognostic factors do not allow for tailoring of treatment to individual patients which could spare many side effects for over-treated women and reduce the number of recurrences in under-treated patient groups.

Molecular studies also indicate the relationship between EC clinical course and several molecular variables, such as: condition of hormonal receptors (ER, PR), ARID1A and c-MET linked to poor prognosis, metastases and mutations in MMR genes detected in Lynch syndrome [17-21]. Recent published studies indicated a relationship between Nesfatin-1 (NESF-1) with its precursor, NUCB2-nucleobindin 2, and the clinical course of EC $[22,23]$. NESF-1 is associated with obesity and diabetes type II (main risk factors for EC) and participates in the regulation of hunger and fat storage, it is associated with insulin resistance and glucose homeostasis.

The aim of this study was to estimate the expression of NESF-1, MLH1 and MLH2 belonging to MMR, C-MET and ARID1A in two types of endometrial cancer and determine relationships between the mentioned factors.

\section{MATERIAL AND METHODS}

This was a multi-centre, retrospective study. The investigated material involved archival, histological preparations, obtained from uterine endometrial cancer samples taken from patients primarily treated surgically between 2007-2014.

All 146 patients included in this retrospective study were monitored for the entire five to eight-year period from diag- nosis of EC through the duration of their treatment and subsequent observation. In order to fulfil the selection criteria for the study, patients must have been diagnosed at one of the centres participating in the study. Only patients where information on FIGO grading and histology were complete, who underwent treatment for EC at the centre where they were originally diagnosed and then monitored for the stated study period by practitioners at that specific centre were included in this study.

Out of a total of 146 patients, 38 (26\%) patients were diagnosed at stage IA according to FIGO, 36 patients (24.7\%) at stage IB, 37 patients (25.4\%) at stage II, 22 (15\%) at stage III and $13(8.9 \%)$ at stage IV.

In the studied group, 115 patients were diagnosed with type I EC (78.8\%), in 31 type II EC (21.2\%) was detected, this included 18 serous types (12.3\%), 11 clear cell types and 2 mucinous types (1.4\%).

In 38 patients (25\%), endometrial cancer manifested a high histological maturity (G1), in 59 patients (38.8\%) it displayed an intermediate histological differentiation (G2) while in 55 patients (36.2\%) undifferentiated tumors were identified (G3) (Tab. 1).

\begin{tabular}{|c|c|}
\hline Clinical Staging acc. to FIGO & Number of patients \\
\hline \multicolumn{2}{|c|}{ Endometrioid adenocarcinoma $(n=115)$} \\
\hline IA & 33 \\
\hline IB & 32 \\
\hline ॥ & 27 \\
\hline III & 14 \\
\hline IV & 9 \\
\hline \multicolumn{2}{|c|}{ Serous adenocarcinoma G3 $(n=18)$} \\
\hline IA & 4 \\
\hline IB & 3 \\
\hline ॥ & 7 \\
\hline III & 4 \\
\hline \multicolumn{2}{|c|}{ Clear cell adenocarcinoma G3 $(n=11)$} \\
\hline IA & 1 \\
\hline IB & 1 \\
\hline$\|$ & 2 \\
\hline III & 4 \\
\hline IV & 3 \\
\hline \multicolumn{2}{|l|}{ Mucinous adenocarcinoma $(n=2)$} \\
\hline$\|$ & 1 \\
\hline IV & 1 \\
\hline Grading & Number of patients \\
\hline \multicolumn{2}{|c|}{ Endometrioid adenocarcinoma $(n=115)$} \\
\hline G1 & 36 \\
\hline G2 & 56 \\
\hline G3 & 23 \\
\hline
\end{tabular}


The mean age of the entire patient group studied was 65.3; in women with endometrioid cancer the mean age was 64.7 (34-83) and in women with non-endometrioid cancer it was $67.6(40-83)(p>0.05)$.

The tissue material was fixed in $10 \%$ buffered formalin, $\mathrm{pH} 7.4$ and placed in a processor. The neoplastic tissue was embedded in paraffin at $60^{\circ} \mathrm{C}$ using standard histopathological techniques. The paraffin blocks were sliced in a microtome to 4-5 um thick sections, placed on adhesive glass slides and left for one hour at a temperature of $60^{\circ} \mathrm{C}$. In the study, the immunohistochemical method - DAKO Envision ${ }^{\mathrm{TM}}$ Flex+ (Dako system, Dako, Santa Clara, USA) was applied. Antigens were detected in the paraffin sections using the Target Retrieval Solution, high $\mathrm{pH}$, DAKO in the PT-link (Dako, Santa Clara, USA) at a temperature of $97^{\circ} \mathrm{C}$, for $20 \mathrm{~min}$.

Nesfatin-1 was estimated using Nesfatin-1/Nucleobinding-2 Antibody (Novus Biologicals, Littleton, USA, NBP187383). In order to detect antigens present in the tissue material antibodies were used against ARID1A (Novus Biological, Littleton, USA, NBP1-88932), Met (Santa Cruz Biotechnology, Santa Cruz, USA, clone C-12, MLH1 (Leica NCL-L, Buffalo Grove, USA. Clone E-305), MSH2 (Invitrogen, Carlsbad, USA, clone FE11).

The intensity of NESF-1, ARID1A, c-MET, MLH1, $\mathrm{MSH} 2$ staining was estimated using a 4-degree scale: no reaction

+1 to 50 immunopositive cells (cell nuclei or the cytoplasm) ++50 to 75 immunopositive cells

+++75 to 100 immunopositive cells per 10 visual fields.

A positive reaction was accepted in the case of preparations manifesting ++ or +++ staining.
Statistical calculations were made using the Mann-Whitney, Kruskall-Wallis and Spearman's tests (STATISTICA StatSoft Inc, USA). Statistical significance was concluded when $\mathrm{p}$ was less than 0.05 .

\section{RESULTS}

A high expression of NESF-1, MLH1, MSH2, c-MET and ARID1A was detected, respectively, in 53.1\%, 57.7\%, 47.3\%, $88.7 \%$ and $48.2 \%$ patients with endometrial cancer.

The immunohistochemical reaction with antibodies against NESF-1 and c-MET was seen in the cytoplasm; MLH1, MSH2 and ARID1A manifested nuclear localization.

\section{Histopathological type}

In respect to histopathological diagnosis the patients formed two groups: endometrioid type $(n=115)$ and non-endometrioid type $(n=31)$, which included patients with serous, clear cell and mucinous cancers.

Positive expression of nesfatin was determined in $50.99 \%$ of patients with type ECI and in $61.3 \%$ of patients with type II EC. No significant difference was disclosed between the groups $(p=0.410)$. MLH1 expression was statistically lower in type EC I in comparison to type II EC (51.9\% vs $77.4 \%, p=0.013)$. No significant difference was disclosed in the expression of MSH2, c-MET and ARIDIA between the two subgroups $(p<0.05)$ (Fig.1).

\section{Clinical stage according to FIGO}

Patients with endometrial cancer were subdivided depending on clinical stage into early stage (IA) and later stage of the disease (IB-IV). In the entire group of patients with endometrial cancer, no relationship was detected between intensity of NESF-1 expression and the stage of clinical ad-

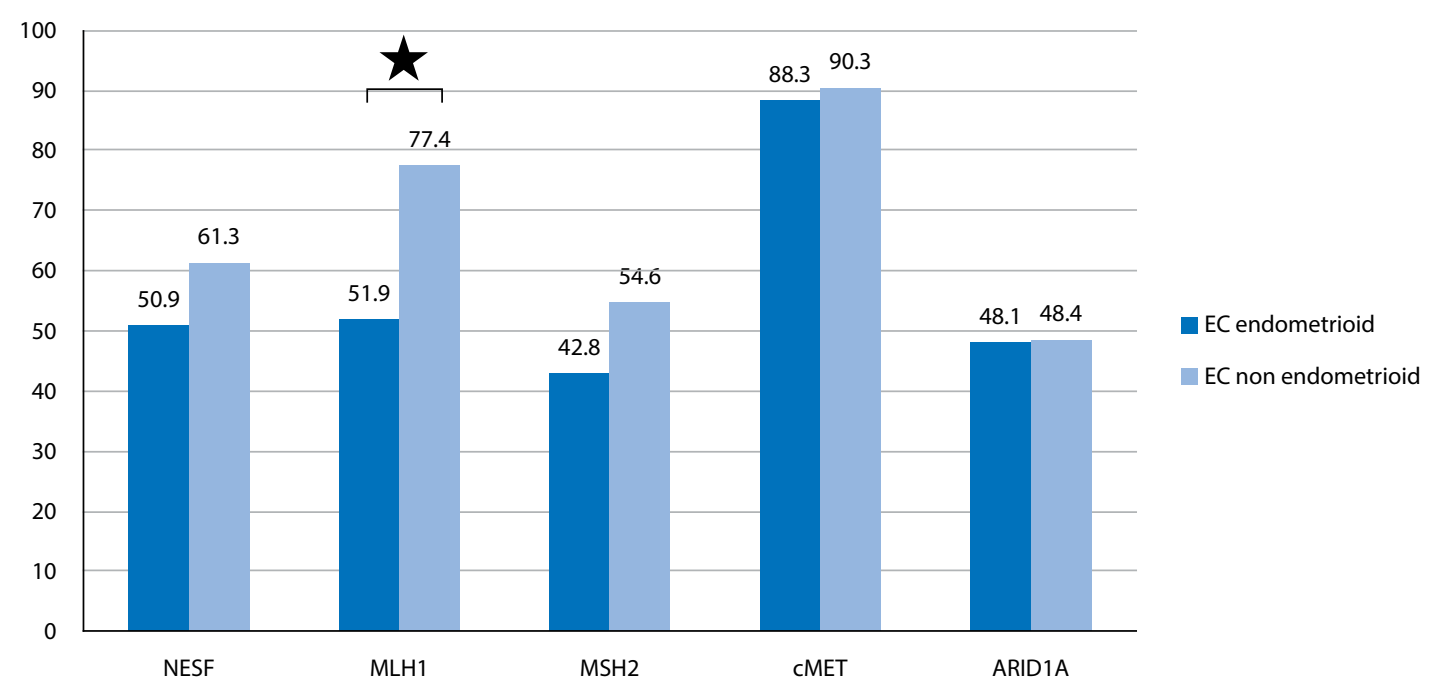

Figure 1. Percentage of endometrial cancer cases manifesting a high expression of a given protein in endometrioid and non-endometrioid cancer cells; $\star p<0.05$ 
vancement of the disease $(p=0.382)$. Also no such relationship was detected for MLH1, MSH2, c-MET and ARIDIA.

\section{Grading}

Among all the analyzed patients no relation was detected between the level of NESF-1 expression and histological grade of cancer differentiation ( $p=0.3145$ ). Furthermore, no such relationship was detected for MLH1, MSH2, c-MET and ARID1A ( $p>0.05$ ) (Fig. 2, 3). The group of patients with type IEC with grade 1 manifested a statistically higher expression of nesfatin than the patients with histologically less mature cancer (G1 68.97\% vs G2 + G3 45.45\%, p = 0.0487). The relationship between the level of studied protein expression and histological grading of non-endometrioid cancers was not analyzed since the latter by definition are poorly differentiated cancers (G3).

\section{Correlation between evaluated proteins}

Among all the studied patients with ECs, a high expression of NESF-1 correlated with a high expression of the MLH1 protein $(p=0.039)$. No relationship was detected between the level of NESF-1 expression and MSH2, c-MET and ARID1A in the entire population of patients with endometrial cancer $(p=0.43, p=0.24, p=0.4158)$.

\section{DISCUSSION}

NESF-1, the amino acid derivative of NUCB2 carries prognostic significance in type I EC; its immunoreactiv-

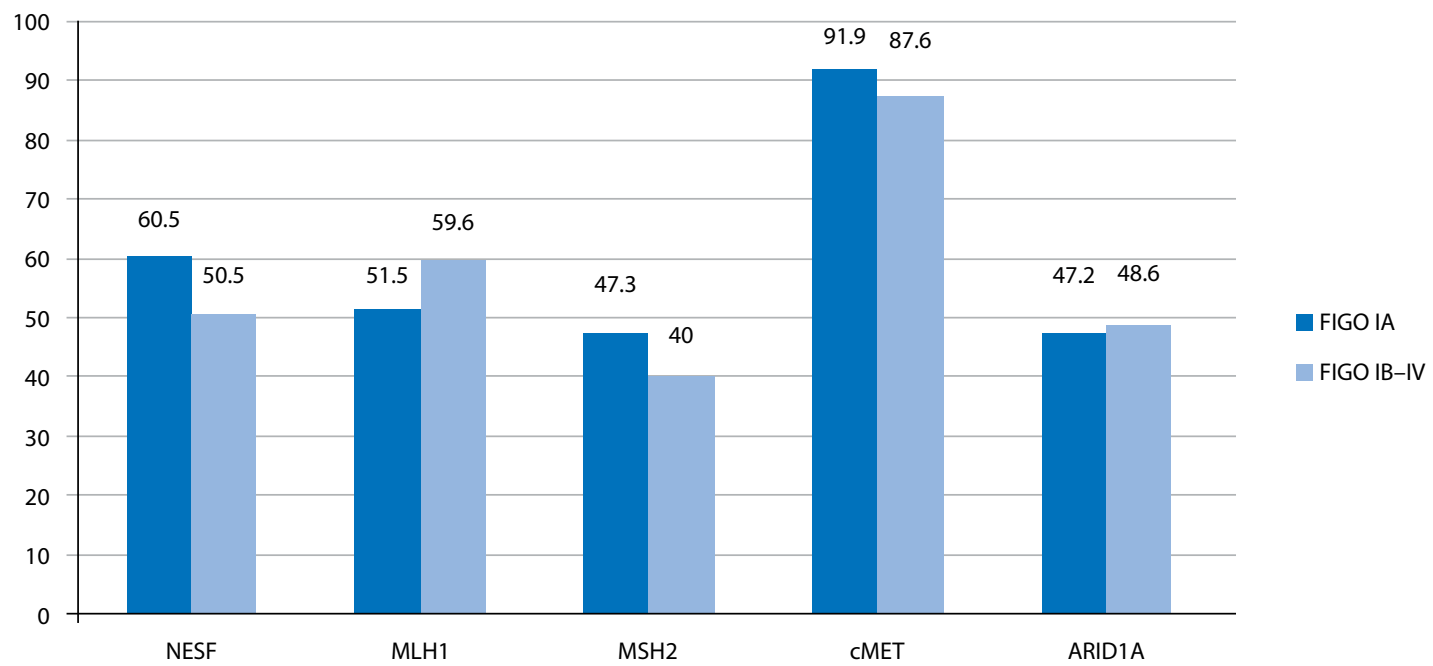

Figure 2. Percentage of endometrial cancer cases manifesting a high expression of a given protein in relation to the clinical stage

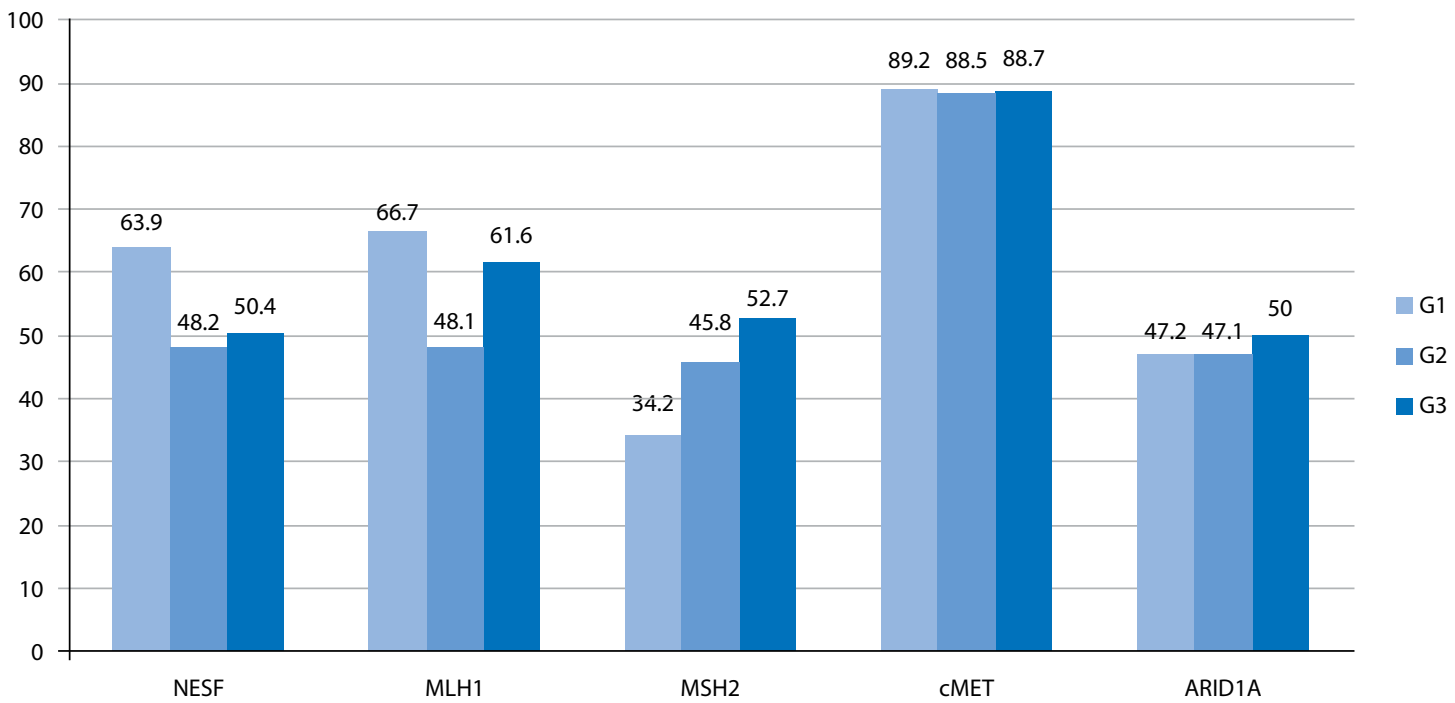

Figure 3. Percentage of endometrial cancer cases in the entire population of patients manifesting high expression of a given protein in relation to histopathological grading 
ity correlated with an augmented risk of relapse and poor clinical course [23]. In the presented study, two groups of patients were analysed — women with endometrial cancer endometrioid type (ECI) and non-endometrioid (EC II). The expression of NESF-1 was more pronounced in type II EC (61.3\%) with a poorer prognosis compared to type I EC (NESF-1 expression in $50.9 \%$ cells). However, no statistically significant values were determined $(p=0.410)$. The evaluation of expression in various grades of histological grading determined that patients with type I EC at G1 manifested a higher expression of NESF-1 than those with type I EC at both $\mathrm{G} 2$ and $\mathrm{G} 3$ (G1 - 68.97\% compared to $\mathrm{G} 2$ and $\mathrm{G} 3-50.4 \%, \mathrm{p}=0.0487$ ). Similar results were observed in studies of Takagi et al. [23] in type IEC, though no statistical significance was identified (in $\mathrm{G} 1$ cancers the proportion of positive reaction was higher than in $\mathrm{G} 2$ and $\mathrm{G} 3$ ).

Mutations in MLH1 and MSH2 linked with Lynch syndrome carry a risk of developing type I EC. In the group of patients presented in this study, the expression of MLH1 protein was significantly higher in type II EC. Among all 146 patients, a high expression of NESF-1 correlated with a high expression of MLH1 $(p=0.039)$, which seems to be slightly controversial. In the literature, a high expression of NESF-1 was linked to poorer prognosis, while MLH1, associated with type I EC, was linked to better prognosis [2, 23, 24]. Dividing the patients according to the histological type of the tumor, a tendency was noted for a high expression of NESF-1 in patients with a high expression of MSH2 ( $p=0.0596)$. This might indicate that despite the normal function of the MMR protein group, a high expression of NESF-1 is significant for prognosis in the patient group.

c-Met, also called tyrosine-protein kinase Met, is proto-oncogene tyrosine kinase, located on chromosome 7q21-31. Physiologically, it is essential for the disruption of cadherin-based cell-cell contacts and subsequent cell motility which takes place is embryonic development, organogenesis and wound healing [25]. It activates a wide range of different cellular signalling pathways, including those involved in proliferation, motility, migration and invasion. In many human primary tumours, amplification of the c-MET gene, with consequent protein overexpression and kinase activation, has been found. In our study, the expression of c-MET was most pronounced among the studied parameters: it was noted in over $80 \%$ of all tissues in both type I and type II ECs. No relationship was detected between the expression of c-MET and clinical stage or grading, although in the literature there is a correlation between its expression and metastatic disease and poor prognosis [26, 27].

$A R I D 1 A$ is located on chromosome $1 \mathrm{p} 36.11$ and encodes protein ARID1A, an important member of the SWI/SNF complex. This complex is responsible for chromatin remodelling, which regulates gene expression depending on changes in chromatin structures, and participates in replication, transcription and repair processes [28]. Dysfunction in the mechanism leads to carcinogenesis mainly via PI3K/AKT pathway. Mutations in ARID1A are found in many cancers and are common in gynaecological cancers such as clear cell and endometrioid ovarian cancer, but also in around $40 \%$ of endometrioid EC [29-31]. In our material, expression of ARID1A protein was detected in around $48 \%$ of all EC patients.

No differences in the expression of ARID1A protein were revealed in our study between clinical stages and histological grades. No relationship was also detected between ARID1A expression and the studied proteins (NESF-1, MLH1, MSH2, c-MET).

In both subgroups of patients, no other relationships were detected between the levels of expression of NESF, MLH1, MSH2, C-MET and AR1D1A.

\section{CONCLUSIONS}

In this study on protein expression, EC tissues manifested (although insignificantly) an elevated expression of NESF-1 in type II EC. In type I EC, NESF-1 expression was significantly higher in $\mathrm{G} 1$ in comparison to $\mathrm{G} 2$ and $\mathrm{G} 3$ combined. A significantly lower expression of MLH1 was demonstrated in type I EC. The most pronounced expression involved c-MET in all EC I and EC II tissues (in over $80 \%$ of cases). A tendency was detected for a high expression of NESF-1 in patients with type II EC, who also exhibited a high expression of $\mathrm{MSH} 2$.

\section{REFERENCES}

1. Globocan 2012 Estimated Cancer Incidence, Mortality and Prevalence Worldwide in 2012. http://globocan.iarc.fr (16.01.2015).

2. Colombo N, Creutzberg C, Amant F, et al. ESMO-ESGO-ESTRO Endometrial Consensus Conference Working Group. ESMO-ESGO-ESTRO Consensus Conference on Endometrial Cancer: Diagnosis, Treatment and Follow-up. Int J Gynecol Cancer. 2016; 26(1): 2-30, doi: 10.1097/IGC.0000000000000609, indexed in Pubmed: 26645990.

3. Murali R, Soslow RA, Weigelt B. Classification of endometrial carcinoma: more than two types. Lancet Oncol. 2014; 15(7): e268-e278, doi: 10.1016/S1470-2045(13)70591-6, indexed in Pubmed: 24872110.

4. Schmeler KM, Soliman PT, Sun CC, et al. Endometrial cancer in young, normal-weight women. Gynecol Oncol. 2005; 99(2): 388-392, doi: 10.1016/j.ygyno.2005.06.029, indexed in Pubmed: 16051336.

5. Bokhman JV. Two pathogenetic types of endometrial carcinoma. Gynecol Oncol. 1983; 15(1): 10-17, doi: 10.1016/0090-8258(83)90111-7, indexed in Pubmed: 6822361.

6. Setiawan VW, Yang HP, Pike MC, et al. Australian National Endometrial Cancer Study Group. Type I and II endometrial cancers: have they different risk factors? J Clin Oncol. 2013; 31(20): 2607-2618, doi: 10.1200/JCO.2012.48.2596, indexed in Pubmed: 23733771.

7. Cossio SL, Koehler-Santos P, Pessini SA, et al. Clinical and histomolecular endometrial tumor characterization of patients at-risk for Lynch syndrome in South of Brazil. Fam Cancer. 2010; 9(2): 131-139, doi: 10.1007/s10689-009-9297-x, indexed in Pubmed: 19821155.

8. Huang M, Djordjevic B, Yates MS, et al. Molecular pathogenesis of endometrial cancers in patients with Lynch syndrome. Cancer. 2013; 119(16): 3027-3033, doi: 10.1002/cncr.28152, indexed in Pubmed: 23760948.

9. Attias-Geva Z, Bentov I, Kidron D, et al. p53 Regulates insulin-like growth factor-I receptor gene expression in uterine serous carcinoma and predicts responsiveness to an insulin-like growth factor-I receptor-directed 
targeted therapy. Eur J Cancer. 2012; 48(10): 1570-1580, doi: 10.1016/j. ejca.2011.09.014, indexed in Pubmed: 22033326.

10. Togami S, Sasajima Y, Oi T, et al. Clinicopathological and prognostic impact of human epidermal growth factor receptor type 2 (HER2) and hormone receptor expression in uterine papillary serous carcinoma. Cancer Sci. 2012; 103(5): 926-932, doi: 10.1111/j.1349-7006.2012.0224 0.x, indexed in Pubmed: 22329832.

11. Bishop EA, Lengyel ER, Yamada SD, et al. The expression of hepatocyte growth factor (HGF) and c-Met in uterine serous carcinoma. Gynecol Oncol. 2011; 121(1): 218-223, doi: 10.1016/j.ygyno.2010.11.031, indexed in Pubmed: 21168200.

12. Bruchim I, Amichay $\mathrm{K}$, Kidron D, et al. BRCA1/2 germline mutations in Jewish patients with uterine serous carcinoma. Int J Gynecol Cancer. 2010; 20(7): 1148-1153, doi: 10.1111/IGC.0b013e3181ef622d, indexed in Pubmed: 21206239.

13. Brinton LA, Felix AS, McMeekin DS, et al. Etiologic heterogeneity in endometrial cancer: evidence from a Gynecologic Oncology Group trial. Gynecol Oncol. 2013; 129(2): 277-284, doi: 10.1016/j.ygyno.2013.02.023, indexed in Pubmed: 23485770.

14. Park JiY, Nam JH, Kim YT, et al. Poor prognosis of uterine serous carcinoma compared with grade 3 endometrioid carcinoma in early stage patients. Virchows Arch. 2013; 462(3): 289-296, doi: 10.1007/s00428013-1382-8, indexed in Pubmed: 23417747.

15. Kandoth C, Schultz N, Cherniack AD, et al. Cancer Genome Atlas Research Network. Integrated genomic characterization of endometrial carcinoma. Nature. 2013; 497(7447): 67-73, doi: 10.1038/nature12113, indexed in Pubmed: 23636398.

16. Piulats JM, Guerra E, Gil-Martín M, et al. Molecular approaches for classifying endometrial carcinoma. Gynecol Oncol. 2017; 145(1): 200-207, doi: 10.1016/j.ygyno.2016.12.015, indexed in Pubmed: 28040204.

17. Kreizman-Shefer H, Pricop J, Goldman S, et al. Distribution of estrogen and progesterone receptors isoforms in endometrial cancer. Diagn Pathol. 2014; 9: 77, doi: 10.1186/1746-1596-9-77, indexed in Pubmed: 24684970.

18. Sho T, Hachisuga T, Nguyen TT, et al. Expression of estrogen receptor-a as a prognostic factor in patients with uterine serous carcinoma. Int J Gynecol Cancer. 2014; 24(1): 102-106, doi: 10.1097/IGC.0000000000000029, indexed in Pubmed: 24335662

19. Takeda T, Banno K, Okawa R, et al. ARID1A gene mutation in ovarian and endometrial cancers (Review). Oncol Rep. 2016; 35(2): 607-613, doi: 10.3892/or.2015.4421, indexed in Pubmed: 26572704.
20. Li M, Xin X, Wu T, et al. HGF and c-Met in pathogenesis of endometrial carcinoma. Front Biosci (Landmark Ed). 2015; 20(8): 635-643, indexed in Pubmed: 25553470.

21. Bischoff J, Ignatov A, Semczuk A, et al. hMLH1 promoter hypermethylation and MSI status in human endometrial carcinomas with and without metastases. Clin Exp Metastasis. 2012; 29(8): 889-900, doi: 10.1007/s10585-012-9478-0, indexed in Pubmed: 22552371.

22. Markowska A, Marszałek A, Szarszewska M, et al. Nesfatin-1 as a potential marker in endometrial and ovarian carcinomas. Post Biol Komórki. 2016; 43(3): 435-439.

23. Takagi K, Miki Y, Tanaka S, et al. Nucleobindin 2 (NUCB2) in human endometrial carcinoma: a potent prognostic factor associated with cell proliferation and migration. Endocr J. 2016; 63(3): 287-299, doi: 10.1507/endocrj.EJ15-0490, indexed in Pubmed: 26842712.

24. Sasada S, Yunokawa M, Takehara Y, et al. Baseline risk of recurrence in stage I-II endometrial carcinoma. J Gynecol Oncol. 2018; 29(1): e9, doi: 10.3802/jgo.2018.29.e9, indexed in Pubmed: 29185267.

25. Corso S, Comoglio PM, Giordano S. Cancer therapy: can the challenge be MET? Trends Mol Med. 2005; 11(6): 284-292, doi: 10.1016/j.molmed.2005.04.005, indexed in Pubmed: 15949770.

26. Zhuang $X P$, Jin $W W$, Teng $X D$, et al. c-Met and RON expression levels in endometrial adenocarcinoma tissue and their relationship with prognosis. Eur J Gynaecol Oncol. 2015; 36(3): 255-259, indexed in Pubmed: 26189249.

27. Li M, Xin X, Wu T, et al. HGF and c-Met in pathogenesis of endometrial carcinoma. Front Biosci (Landmark Ed). 2015; 20(8): 635-643, indexed in Pubmed: 25553470.

28. Clapier CR, Cairns BR. The biology of chromatin remodeling complexes. Annu Rev Biochem. 2009; 78: 273-304, doi: 10.1146/annurev. biochem.77.062706.153223, indexed in Pubmed: 19355820.

29. Mao TL, Ardighieri L, Ayhan A, et al. Loss of ARID1A expression correlates with stages of tumor progression in uterine endometrioid carcinoma. Am J Surg Pathol. 2013; 37(9): 1342-1348, doi: 10.1097/PAS.0b013e3182889dc3, indexed in Pubmed: 24076775.

30. Wiegand KC, Shah SP, Al-Agha OM, et al. ARID1 A mutations in endometriosis-associated ovarian carcinomas. N Engl J Med. 2010; 363(16): 15321543, doi: 10.1056/NEJMoa1008433, indexed in Pubmed: 20942669.

31. Guan B, Mao TL, Panuganti PK, et al. Mutation and loss of expression of ARID1A in uterine low-grade endometrioid carcinoma. Am J Surg Pathol. 2011; 35(5): 625-632, doi: 10.1097/PAS.0b013e318212782a, indexed in Pubmed: 21412130. 